\title{
Uso da Monitorização Contínua de Glicose (CGMS) por quatro (96 horas) ou cinco (120 horas) Dias em Pacientes com DM1: Existe Vantagem?
}

\author{
RESUMO
}

\begin{abstract}
Objetivo: Avaliar a acurácia, as complicações e o impacto no controle glicêmico de pacientes com diabetes melito tipo 1 (DM1) submetidos ao uso da monitorização contínua de glicose (CGMS) por quatro e cinco dias. Métodos: Estudamos 36 pacientes $\mathrm{DM} 1(44,5 \% \mathrm{M} / 55,5 \% \mathrm{~F})$, divididos em três grupos, com idade, tempo de DM e A1c semelhantes $(p<0,05)$, submetidos ao CGMS por $72 \mathrm{~h}(\mathrm{G} 1), 96 \mathrm{~h}(\mathrm{G} 2)$ e $120 \mathrm{~h}$ (G3). Foram analisados: GC média e pelo sensor, número de leituras, coeficiente de correlação, mediana da diferença absoluta (MAD\%); complicações (trauma, infecção, desconexão, abandono); hiperglicemia pós-prandial (HPP) e hipoglicemia assintomática $(<70 \mathrm{mg} / \mathrm{dl})$. Os níveis de A1c após três meses e um ano foram determinados nos três grupos. Resultados: Não houve diferença técnica entre os grupos: coeficiente de correlação $>0,79$ e MAD\% $<28 \%(p<0,01)$ em $95 \%$. O uso do sensor por $>72 \mathrm{~h}$ não se associou com erro de sinal, desconexão, trauma, alarmes ou infecção local $(p<0,01)$. Verificou-se alta correlação entre GC média e pelo sensor, nos três grupos $(p=0,01)$. A hipoglicemia silenciosa foi mais duradoura (min.) à noite versus dia $(p=0,05)$, em todos os grupos. A HPP foi estatisticamente mais detectada nos grupos 1 e 3 . Observou-se redução significante da A1c três meses após a CGMS no G1 (72 h) e G3 (120 h) ( $p<0,001$ e p = 0,002, respectivamente), que se manteve após um ano ( $p<0,001$ e $p=0,047$, respectivamente). Conclusão: Evidenciamos alta acurácia/eficácia técnica com baixo índice de complicações em pacientes submetidos ao CGMS por $96 \mathrm{~h}$ ou $120 \mathrm{~h}$. Não verificamos benefícios em relação à CGMS por $72 \mathrm{~h}$ quanto à redução da $A 1 \mathrm{c}$ em curto (três meses) e médio (um ano) prazo. O sensor CGMS pode ser utilizado por $>72 \mathrm{~h}$, sem prejuízo técnico, mas sem grandes benefícios do ponto de vista clínico, para pacientes com DM1. (Arq Bras Endocrinol Metab 2008; 52/3:499-505)
\end{abstract}

Descritores: Sistema de monitorização contínua da glicose; Diabetes melito tipo 1

\section{ABSTRACT}

Continuous Glucose Monitoring System (CGMS) in Type 1 Diabetic Patients During 4 (96h) or 5 (120h) Days: There Is Advantage?

Background: To evaluate the accuracy, complications and impact in glycemic control in type 1 diabetic patients (DM1) submitted to 4 or 5 days of CGMS. Methods: We studied $36 \mathrm{DM} 1$ patients $(44.5 \% \mathrm{M} / 55.5 \% \mathrm{~F})$, in three groups without no difference about age, DM duration and $A 1 c$ levels $(p<0.05)$, submitted to $72 \mathrm{~h}(\mathrm{G} 1), 96 \mathrm{~h}(\mathrm{G} 2)$ and $120 \mathrm{~h}$ (G3) CGMS profile. It were analyzed: capillary glycemia (CG) and mean CGMS sensor's glycemic value; correlation coefficient, median absolute percent difference (MAD\%), number of sensor reading, complications (trauma, local infection, disconnection, dropped), postprandial hyperglycemia, unrecognized hypoglycemia $(<70 \mathrm{mg} / \mathrm{dl})$. A1c levels were measured at the start (1 month before) and after 3 and 12 months in each group.

\section{artigo original}

\author{
FREDERICO F. R. MAIA \\ LeVIMAR R. ARaújo
}

Departamento de Fisiologia da

Faculdade de Ciências Médicas de Minas Gerais (FCMMG);

Clínica de Endocrinologia e Metabologia do Hospital Universitário São José da FCMMG, Belo Horizonte, MG, Brasil.

Recebido em 03/09/2007

Aceito em 17/12/2007 
Results: No technical difference were observed into 3 groups: correlation coefficient $>0.79$ and MAD $<28 \%$ in $95 \%$ ( $p<0.01)$. The use of CGMS sensor more than $72 \mathrm{~h}$ was not related to signal error, trauma, local infection or disconnection. The mean capillary glucose values showed no difference by glucose CGMS sensor $(p=0.01)$ in all groups. The nighttime episodes of hypoglycemia lasted longer ( $\mathrm{min}$ ) than daytime episodes in all groups $(p=0.05)$. The postprandial hyperglycemia was statistically identified in groups 1 and 3 . This data showed significant decreased A1c level three months after the CGMS in G1 (72h) and G3 (120h) ( $<0.001$ and $p=0.002$, respectively), which sustained after 1 year ( $p<0.001$ e $p=0.047$, respectively). Conclusions: The CGMS showed to be a very safety method, with high accuracy/technical efficacy in patients undergoing $96 \mathrm{~h}$ and $120 \mathrm{~h}$ of CGMS. We do not observed advantages in the use of CGMS during $96 \mathrm{~h}$ or $120 \mathrm{~h}$ against $72 \mathrm{~h}$ in decrease A1c levels after 3 and 12 months. It is possible the use of CGMS $>72 \mathrm{~h}$, with no technical damage. However, we do not observed significant clinical benefits of this conduct in DM1 patients. (Arq Bras Endocrinol Metab 2008; 52/3:499-505)

Key-words: Continuous glucose monitoring system; Type 1 diabetes mellitus

\section{INTRODUÇÃo}

USO DO SISTEMA DE monitorização contínua de glicose (CGMS) tem se tornado cada vez mais comum em nosso meio, na busca de um tratamento intensivo, com melhor ajuste terapêutico e redução de glicohemoglobina (Alc) em pacientes com diabetes melito tipo 1 (DMl). A glicemia capilar no monitoramento da doença é um grande avanço, iniciada nos anos 1980, realizada com amostras coletadas em ponta de dedo, imprescindível no controle do DMl (1). A dor dificulta a realização da glicemia capilar em "ponta de dedo" por causa das inúmeras terminações nervosas nesse local. A monitorização do DMl pela glicemia capilar (GC) fornece apenas dados intermitentes do controle metabólico, impedindo a visão completa do perfil glicêmico do paciente (1).

Com o CGMS tem sido possível avaliar com exatidão as variações glicêmicas ao longo do dia, com intervenção terapêutica mais eficaz e redução da Alc $(2,3)$. Diversos estudos têm demonstrado a eficácia desse método de monitoramento da glicose, com repercussões importantes sobre o perfil metabólico dos pacientes (4-6). Verifica-se maior detecção de hiperglicemias pósprandiais (HPP), inclusive em adultos, idosos e crianças, hipoglicemia noturna e silenciosa, bem como impacto na redução da Alc no período de três a seis meses após o uso da monitorização contínua por 72 h (7-9).

Conforme recomendação do fabricante, o sensor do CGMS deve ser idealmente utilizado por período de
72 h (três dias). A revisão da literatura indexada no período de 2000 a 2007 evidenciou apenas um estudo com uso do sensor CGMS por sete dias, após ter expirado o tempo de validade do sensor, em indivíduos não-diabéticos (10). Na prática clínica diária, observase o uso do CGMS por períodos variados de 24 h à 120 $\mathrm{h}$, realizado tanto por indústrias de laboratórios quanto por equipes em clínicas especializadas, sem critérios bem estabelecidos na literatura atual. Não há conhecimento sobre o impacto do uso do CGMS por 24, 48, 96 ou $120 \mathrm{~h}$ em pacientes com DMl em relação às vantagens clínicas (redução de Alc, hipoglicemia silenciosa e excursões glicêmicas), tampouco quanto aos riscos e complicações nesses casos.

Diante da escassez de dados científicos sobre o tema, avaliamos um grupo de pacientes com DMl, submetidos ao CGMS por 72, 96 e $120 \mathrm{~h}$. Buscou-se avaliar a acurácia/eficácia técnica do método, as complicações e o impacto no controle glicêmico em curto e médio prazos (três e 12 meses, respectivamente) de pacientes com DMl submetidos ao CGMS por três, quatro e cinco dias.

\section{CASUÍSTICA E MÉTOdOS}

Foi realizado um estudo retrospectivo, com análise qualitativa e quantitativa, de pacientes com DMl, atendidos no Instituto Avançado em Diabetes e Endocrinologia (Inade), Belo Horizonte, MG, no período de maio de 2006 a julho de 2007. 


\section{Pacientes}

Foram avaliados 36 pacientes com DMl, divididos em três grupos $(n=12)$, com idade, tempo de DMl e Alc semelhantes (Tabela $1 ; \mathrm{p}<0,05$ ), submetidos ao CGMS por $72 \mathrm{~h}$ (G1), 96 h (G2) e 120 h (G3); sendo 44,5\% do sexo masculino e $55,5 \%$ do sexo feminino. A idade variou de 5 a 46 anos. Não houve restrição quanto a idade, sexo ou tempo de diabetes.

Tabela 1. Características dos pacientes com DM1 submetidos ao CGMS por 72,96 e $120 \mathrm{~h}$.

\begin{tabular}{lcccc}
\hline Variáveis & $\mathbf{N}$ & $\mathbf{G 1}(\mathbf{7 2} \mathbf{h})$ & $\mathbf{G 2}(\mathbf{9 6} \mathbf{h})$ & $\mathbf{G 3}(\mathbf{1 2 0} \mathbf{h})$ \\
\hline ldade (anos) & 12 & $22,6 \pm 9,5$ & $23,8 \pm 14,3$ & $23,8 \pm 13,7$ \\
\hline $\begin{array}{l}\text { Tempo de DM1 } \\
\text { (anos) }\end{array}$ & 12 & $11,3 \pm 8,8$ & $11,1 \pm 8,9$ & $8,67 \pm 7,7$ \\
\begin{tabular}{l} 
Alc prévia (\%) \\
\hline
\end{tabular} & 12 & $9,3 \pm 1,5$ & $9,3 \pm 1,6$ & $8,6 \pm 1,4$ \\
\hline
\end{tabular}

\section{Sensor de glicose (CGMS)}

A monitorização contínua da glicose (CGM) foi realizada pelo monitor Medtronic/Northridge, CA. A glicose é mensurada com base na reação eletroquímica da enzima glicose oxidase presente no sensor com a glicose do fluido intersticial. Os valores variam entre $40 \mathrm{e}$ $400 \mathrm{mg} / \mathrm{dl}$ e são captados a cada 10 segundos, com o registro da média desses valores a cada cinco minutos, no total de 288 medidas ao dia e 864 medidas durante o exame $(72 \mathrm{~h})$, conforme recomendação do fabricante. Sessenta minutos após a instalação, a corrente elétrica em nanoamperes gerada é convertida em glicose, após a inclusão de um valor de glicemia capilar no monitor. Ao final do registro, o sensor é descartado e os dados armazenados são transferidos ao software, para análise e interpretação. Todos os pacientes utilizaram sensor de CGMS dentro do prazo de validade estipulado pelo fabricante.

\section{MÉTODOS}

Todos os pacientes foram submetidos previamente a orientações básicas de funcionamento do monitor, pelo mesmo examinador, técnica de manuseio (registro de eventos e glicemia no monitor), cuidados gerais e preenchimento do "diário do paciente". Os pacientes foram orientados a realizar e incluir o mínimo de quatro valores de GC no monitor por dia. A monito- rização foi realizada pelos próprios pacientes, com glicosímetro digital (Accu-Chek Active; Roche Diagnosis), lancetador Accu-Chek Softclix ${ }^{\circledast}$ Pro e respectivas lancetas. A primeira GC foi realizada após 60 minutos do início da monitorização contínua.

As variáveis técnicas foram: glicemia capilar média (GCM); glicose média pelo sensor CGMS; coeficiente de correlação (\%) entre GC e glicose pelo sensor; mediana da diferença percentual absoluta (MAD\%); número total de leituras; presença de complicações (trauma, infecção local, sangramento, aversão psicológica, inoperabilidade técnica, desconexão, outros "alarmes"); e abandono.

As variáveis clínicas foram: excursões da glicose detectadas pelo sensor CGMS e pela GC; presença de HPP e hipoglicemia silenciosa (durante o dia e a noite); e níveis de Alc antes (um mês), três e 12 meses após o CGMS.

O coeficiente de correlação entre glicemia capilar/glicose registrada pelo sensor e a MAD\% fundamentaram-se na análise do programa Medtronic, definidos como ideal com valores de $>0,79 \%$ e $<28 \%$, respectivamente. A MAD\% foi determinada pela mediana da diferença entre o valor absoluto da glicose captada pelo sensor menos o valor de glicose captado pelo glicosímetro. O número de leituras pelo sensor foi considerado significativo quando acima de $80 \%$ da captação máxima.

As excursões glicêmicas identificadas pela GC foram fundamentadas na informação ("diário") do paciente e na análise do glicosímetro e comparadas com as excursões da glicose detectadas pelo sensor. Consideraram-se excursões glicêmicas valores de glicemia $<70 \mathrm{mg} / \mathrm{dl}$ (hipoglicemia) e $>180 \mathrm{mg} / \mathrm{dL}$ (hiperglicemia).

A presença de HPP foi definida para valores de glicemia superiores a $140 \mathrm{mg} / \mathrm{dl}$, duas horas após o almoço. A hipoglicemia noturna assintomática foi definida por glicemia $<70 \mathrm{mg} / \mathrm{dl}$, no período entre $23 \mathrm{~h} \mathrm{e} 7 \mathrm{~h}$.

Os níveis de glicohemoglobina (Alc) foram determinados antes, três e 12 meses após o CGMS. Os valores de Alc foram estabelecidos pelo método HPLC, com valores de referência entre $4,3 \%$ e $6,9 \%$.

A detecção de complicações teve como base a informação do paciente, durante e após o término do exame. Foram avaliados: trauma durante a aplicação (sangramento, dor), trauma ao CGMS, infecção local, sangramento, aversão psicológica, inoperabilidade técnica, desconexão e outros "alarmes". A tolerância dos pacientes ao CGMS foi fundamentada na taxa de abandono e na necessidade de interrupção do método. 


\section{Estatística}

Os dados foram coletados em uma ficha-padrão e analisados pelo software Minitab, versão 14, por meio do teste qui-quadrado e teste $t$ de Student. Os dados são apresentados como média/desvio-padrão. As variáveis técnicas e clínicas foram comparadas entre os três grupos, considerando $\mathrm{p}<0,05$ para significância estatística.

\section{RESULTADOS}

Quanto às variáveis técnicas, não houve diferença entre os grupos: coeficiente de correlação acima da referência $(>0,79)$ e $\mathrm{MAD} \%<28 \%(\mathrm{p}<0,01)$ em mais de $95 \%$ dos pacientes. O número de leituras do sensor foi $>$ 90\% nos três grupos (Tabela 2), sem diferença significante. Verificou-se alta correlação entre GC média e pelo sensor, independente do tempo de CGMS ( $\mathrm{p}=$ $0,01)$. O uso do sensor por $96 \mathrm{~h}$ ou $120 \mathrm{~h}$ não se associou com erro de sinal, desconexão, trauma, alarmes ou infecção local $(\mathrm{p}<0,01)$.

Quanto às variáveis clínicas, o CGMS detectou mais excursões glicêmicas em relação à GC média $(12,9$ $\pm 5,9$ versus $10,1 \pm 4,9 ; \mathrm{p}=0,01)$ no geral, mais evidente no G3 $(120 \mathrm{~h})(\mathrm{p}=0,018)$. A HPP foi evidenciada em $58,8 \%$ dos casos, com valor médio de $160 \pm 66,2$ $\mathrm{mg} / \mathrm{dl}(\mathrm{VR}<140 \mathrm{mg} / \mathrm{dl})$. Na análise por grupos, a HPP foi mais evidente nos grupos 2 e 3 (Tabela 3). A hipoglicemia noturna foi detectada em $52,8 \%$ dos casos. A hipoglicemia silenciosa (assintomática) foi estatisticamente mais duradoura (mín.) durante o período noturno versus o período diurno $(140,8 \pm 45,5$ versus $67,5 \pm 23,7 ; \mathrm{p}=0,05)$, comprovado também na análise por grupos, sem diferença significante (Tabela 3 ).

Tabela 2. Variáveis técnicas do CGMS por 72,96 e $120 \mathrm{~h}$ em pacientes com DM1.

\begin{tabular}{lccccc}
\hline Variáveis & N & G1 (72 h) & G2 (96 h) & G3 (120 h) & P \\
\hline № leituras (N) & 12 & $766,3 \pm 101,7$ & $1.000,6 \pm 104,2$ & $1.141,5 \pm 104,7$ & NS \\
\hline MAD \% & 12 & $13,8 \pm 6,4$ & $10,1 \pm 4,1$ & $14,3 \pm 4,3$ & NS \\
Coef. correlação & 12 & $0,89 \pm 0,1$ & $0,97 \pm 0,1$ & $0,91 \pm 0,1$ & NS \\
\hline GMS $(\mathrm{mg} / \mathrm{dl})$ & 12 & $207,5 \pm 37,3$ & $204,9 \pm 48,1$ & $169,8 \pm 48,1$ & - \\
GCM (mg/dl) & 12 & $215,1 \pm 50,8$ & $186,5 \pm 43,4$ & $158,7 \pm 46,1$ & - \\
Valor de P & - & $<0,001$ & 0,01 & $<0,001$ & -
\end{tabular}

Coef. correlação = coeficiente de correlação glicose versus sensor estimado pelo CGMS; GCM = glicemia capilar média; GCS = glicose média pelo sensor CGMS; NS: não-significante.

Tabela 3. Variáveis clínicas do CGMS por 72, 96 e $120 \mathrm{~h}$ em pacientes com DM1.

\begin{tabular}{|c|c|c|c|c|c|}
\hline Variáveis & $\mathbf{N}$ & Gl (72 h) & G2 (96 h) & G3 $(120 h)$ & $\mathbf{P}$ \\
\hline Excursão GC & 12 & $8,8 \pm 6,2$ & $12,5 \pm 13,7$ & $16,8 \pm 5,4$ & NS \\
\hline Excursão sensor & 12 & $7,8 \pm 5,0$ & $11,8 \pm 5,1$ & $10,5 \pm 4,5$ & NS \\
\hline Valor de P & 36 & 0,36 & 0,74 & 0,018 & 0,018 \\
\hline HPP (\%) & 12 & $66,7 \%$ & $50 \%$ & $66,7 \%$ & NS \\
\hline Glicose 2 h PP (mg/dl) & 12 & $135,1 \pm 82,6$ & $172,9 \pm 59,5$ & $172,9 \pm 50,8$ & NS \\
\hline HS Dia (min) & 12 & $52,6 \pm 21,2$ & $94,2 \pm 51,2$ & $40,0 \pm 15,8$ & 0,05 \\
\hline HS Noite (min) & 12 & $69,3 \pm 53,9$ & $135,0 \pm 98,4$ & $205,4 \pm 69,9$ & - \\
\hline Alc prévia (\%) & 12 & $9,3 \pm 1,5$ & $9,3 \pm 1,6$ & $8,6 \pm 1,4$ & \\
\hline Alc 3 meses (\%) & 12 & $8,0 \pm 1,2$ & $8,6 \pm 1,3$ & $7,5 \pm 1,2$ & \\
\hline Valor de P & - & $<0,001$ & 0,091 & 0,002 & $<0,001$ \\
\hline Alc 1 ano (\%) & 12 & $8,0 \pm 1,0$ & $8,5 \pm 1,9$ & $7,8 \pm 1,1$ & \\
\hline Valor de P & - & $<0,001$ & 0,107 & 0,047 & $<0,001$ \\
\hline
\end{tabular}

Excursão GC = excursões glicêmicas detectadas por paciente, em média pela glicemia capilar (GC) versus sensor CGMS (< 70 ou > 180 mg/dl); HPP = hiperglicemia pós-prandial; HS = hipoglicemia silenciosa; NS = não-significante. 
A avaliação do controle metabólico (Alc) nos 36 pacientes com DMl após três meses do CGMS evidenciou redução significante da $\mathrm{Alc}(9,12 \pm 1,5$ versus $8,1 \pm 1,3$; $\mathrm{p}<0,001)$. Na análise por grupos, observou-se redução significante da Alc 3 meses após o uso do CGMS no Gl (72 h) e G3 (120 h) (p < 0,001 e p = 0,002, respectivamente), que se manteve após um ano (Tabela 3 ).

Em 91,7\% dos exames, não se observaram complicações durante a utilização do CGMS. O sinal de "erro de glicose" foi o mais comum nesses casos $(8,3 \%)$, sem diferença entre os grupos. Não houve registro de trauma, infecção local ou sangramento durante o estudo. Não houve nenhum caso de aversão psicológica, inoperabilidade técnica ou abandono do método, independente do tempo de CGMS.

\section{DISCUSSÃo}

O estudo realizado buscou demonstrar a acurácia do CGMS em pacientes com DMl, bem como as complicações relacionadas a esse método de monitorização, quando submetidos a período prolongado de uso $(96 \mathrm{~h}$ e $120 \mathrm{~h}$ ). Verificou-se alto índice de correlação entre as medidas captadas pelo sensor CGMS e da glicemia capilar, alta eficácia técnica, associados à tolerância satisfatória pelos pacientes e baixos índices de complicações durante o procedimento.

Quanto à acurácia do CGMS em relação à GC, observou-se alto índice de correlação nesse estudo, o que é corroborado pela literatura (1-3,8-12). O tempo de CGMS acima do habitual ( 96 h ou 120 h) não proporcionou piora da captação do sensor, com alta correlação nos valores de glicose mensurados em pacientes com DMl. Chlup e cols. (10) avaliaram 20 sensores de pacientes não-diabéticos, com 3 a 18 meses do prazo de validade, por sete dias. Verificaram sucesso na captação de sinal pelo sensor em 83,6\% das medidas de glicose. Os parâmetros analisados pelo próprio CGMS (MAD e coeficiente de correlação) permaneceram acima da referência em $100 \%$.

A correlação dos valores da GC e do sensor em 21 pacientes com DMl $(8 \times /$ dia $)$, analisada por DjakourePlatonoff e cols., mostrou coeficiente de correlação de 0,92 , com significância em 93\% dos casos e MAD\% de $25 \pm 2$. Os autores concluíram que o CGMS por $72 \mathrm{~h}$ oferece alta acurácia nas medidas obtidas, sendo método de eleição para determinação do perfil glicêmico do paciente diabético (9), em consonância com Guerci e cols. (11) em 18 adolescentes DMl e Maia e Araújo (1) $(\mathrm{n}=46 \mathrm{DMl})$.

Além do tempo de uso do sensor (idealmente $72 \mathrm{~h}$ ) e do prazo de validade, outra preocupação técnica seria a inclusão do número de GC no monitor do CGMS. O fabricante recomenda o mínimo de $4 \times /$ dia, como utilizado nesse estudo. Uma análise recente de 50 pacientes DMl (10 a 18 anos), utilizou freqüências variadas de GC de 3, 4, 6 e 7 vezes ao dia, em horários diversos, em períodos de maior e menor estabilidade glicêmica. Verificaram maior acurácia no grupo de maior inclusão de GC no monitor $(7 \times /$ dia $)$. No entanto, observaram que a freqüência de inclusão de GC no monitor parece ser menos importante que a realização e inclusão da GC em períodos de maior estabilidade glicêmica, antes de deitar e ao acordar (13).

Quanto à incidência de complicações, o CGMS mostrou-se método bem tolerado pelos pacientes, sem interrupção do exame em nenhum caso, independente do tempo de monitorização. Não houve registro de traumatismo, infecção ou sangramento local em nenhum paciente, em consonância com Chlup e cols. (10). Não foram encontrados dados da literatura internacional em relação ao assunto, o que reforça a importância desses achados em relação ao CGMS em DMl, quando submetidos a períodos prolongados de monitorização ( 4 ou 5 dias).

Quanto ao perfil glicêmico dos pacientes, diversos estudos evidenciam a eficácia desse método na redução da Alc em curto prazo $(1,3,4,7,11,14-16)$. Em adolescentes e crianças com DMl, observou-se eficácia estatisticamente significante do CGMS em promover redução da Alc, bem como ajuste terapêutico e maior educação e motivação do paciente para o tratamento $(2,8,14)$, benefício não comprovado em alguns outros estudos $(17,18)$. Nesse estudo, verificamos redução significante da Alc após três meses nos 36 pacientes acompanhados, sem benefício do maior tempo de monitorização $(120 \mathrm{~h})$ em relação às $72 \mathrm{~h}$ preconizadas. Além desse importante achado, este estudo mostra o benefício prolongado da CGMS mantido por um ano, em pacientes $\mathrm{DMl}$, sem diferença entre três e cinco dias de monitorização. Trata-se do primeiro registro na literatura indexada dos benefícios (redução da Alc), em longo prazo, proporcionados pelo CGMS em pacientes DMl, fato de extrema importância no acompanhamento e no controle desse grupo de pacientes.

Quanto à eficácia do sensor CGMS na identificação de excursões glicêmicas, observamos alta eficácia do 
CGMS em relação à GC $(4 \times /$ dia $)(\mathrm{p}<0,01)$, já comprovado pela literatura, inclusive em idosos institucionalizados $(1,6,7,19)$. A HPP foi identificada em número significativo de pacientes nesse estudo (60\%), sendo mais evidente no grupo de maior período de CGMS (120 h).

Em relação à identificação de episódios de hipoglicemia, alguns estudos evidenciam alta acurácia do CGMS para detecção de hipoglicemias silenciosas $(1,4,8,11,14,20,21)$. Guillod e cols. (22) verificaram hipoglicemia noturna em $67 \%$ de 88 pacientes com DMl, com melhora após seis meses da realização do CGMS. Boland e cols. (20) compararam os efeitos do CGMS versus GC em 56 crianças com DMl, por $72 \mathrm{~h}$. Observou-se superioridade do CGMS na detecção de excursões glicêmicas e hipoglicemia silenciosa $(70 \%)$, corroborado por Maia e Araújo (8). Observou-se maior tempo de hipoglicemia silenciosa durante a noite, de maneira significante, achado presente em todos os grupos, independente do tempo de CGMS $(3,4$ ou 5 dias), como encontrado por Jeha e cols. (2004), em crianças DMl, por três dias (23).

Este estudo mostrou a persistência de alta eficácia técnica do CGMS em pacientes com DMl, com baixo índice de complicações, mesmo quando usado por $96 \mathrm{~h}$ e 120 h, não sendo observadas vantagens significativas da monitorização prolongada $(>72 \mathrm{~h}$ ) do ponto de vista clínico (redução da Alc e detecção de hipoglicemia silenciosa).

Algumas limitações do estudo devem ser enfatizadas: estudo observacional, não randomizado, tratados em unidade de referência. Algumas particularidades, como o entusiasmo dos pacientes com o novo tratamento e o empenho da equipe em educar e obter bons resultados, são capazes de influenciar os resultados e não são passíveis de controle. Contudo, esse trabalho representa a prática clínica real com essa nova terapia, a experiência do dia-a-dia de um grupo de estudos em pacientes com DMl.

\section{CONCLUSÕES}

Evidenciamos alta acurácia/eficácia técnica com baixo índice de complicações em pacientes DMl submetidos ao CGMS por 96 ou $120 \mathrm{~h}$. Não verificamos benefícios em relação à CGMS por $72 \mathrm{~h}$ quanto à redução da Alc em curto (três meses) e médio (um ano) prazos em DMl. Este estudo mostra ser possível utilizar o sensor CGMS por mais de $72 \mathrm{~h}$ (em até cinco dias), sem prejuízo técni$\mathrm{co}$, no entanto sem grandes benefícios do ponto de vista clínico (redução da Alc, detecção de excursões glicêmicas e hipoglicemia silenciosa), para pacientes com DMl, acompanhados por até um ano da monitorização.

\section{REFERÊNCIAS}

1. Maia FFR, Araújo LR. Efficacy of continuous glucose monitoring system (CGMS) to detect postprandial hyperglycemia and unrecognized hypoglycemia in type 1 diabetic patients. Diabetes Res Clin Pract. 2006;75(1):30-4.

2. Kaufman FR. Role of continuous glucose monitoring in pediatric patients. Diabetes Technol Ther. 2000;2:S49-52.

3. Boland EA, Tamborlane VW. Continuous glucose monitoring in youth with type 2 diabetes: overcoming barriers to successful treatment. Diabetes Technol Ther. 2000;2:S53-9.

4. Maia FFR, Araújo LR. Impacto do sistema de monitorização contínua da glicose em pacientes diabéticos. Rev Assoc Méd Bras. 2006;52(6):395-400.

5. Sachedina N, Pickup JC. Performance assessment of the medtronic-minimed continuous glucose monitoring system and its use for measurement of glycaemic control in type 1 diabetic subjects. Diabet Metab. 2003;20(12):1012-5.

6. Maia FFR, Araújo LR. Acurácia, efeitos na terapia insulínica/ controle glicêmico e complicações do sistema de monitorização contínua da glicose (CGMS) em pacientes com diabetes mellitus tipo 1. Arq Bras Endocrinol Metab. 2005;48(4):563-8.

7. Jamali R, Bachrach-Lindström M, Mohseni S. Continuous glucose monitoring system signals the occurrence of marked postprandial hyperglycemia in the elderly. Diabetes Technol Ther. 2005;7:509-15.

8. Maia FF, Araújo LR. Accuracy, utility and complications of continuous glucose monitoring system (CGMS) in pediatric patients with type 1 diabetes. J Pediatr (Rio J). 2005;81:293-7.

9. Djakoure-Platonoff C, Radermercker R, Reach G, Slama G, SeIam JI. Accuracy of the continuous glucose monitoring system in inpatient and outpatient conditions. Diabetes Metab. 2003;29(2 Pt 1):159-62.

10. Chlup R, Jelenová D, Chlupová K, Zapletalová J, Chlupová L, Bartek J. Function and accuracy of glucose sensors beyond their stated expiry date. Diabetes Technol Ther. 2006;8:495-504.

11. Guerci B, Floriot M, Bohme $P$, Durain D, Benichou M, Jellimann $\mathrm{S}$, et al. Clinical performance of CGMS in type 1 diabetic patients treated by continuous subcutaneous insulin infusion using insulin analogs. Diabetes Care. 2003;26(3):582-9.

12. Zhou J, Jia WP, Yu M, Yu HY, Bao YQ, Ma XJ, et al. The reference values of glycemic parameters for continuous glucose monitoring and its clinical application. Zhonghua Nei Ke $\mathrm{Za}$ Zhi. 2007;46:189-92

13. Diabetes Research in Children Network (DirecNet). Evaluation of factors affecting CGMS calibration. Diabetes Technol Ther. 2006;8:318-25.

14. Ludvigsson J, Hanas R. Continuous subcutaneous glucose monitoring improved metabolic control in pediatric patients with type 1 diabetes: a controlled crossover study. Pediatrics. 2003;111(5 Pt 1):933-8.

15. Schaepelynck-Belicar P, Bague P, Simonin G, Lassmann-Vague V. Improved metabolic control in diabetic adolescents using 
the continuous glucose monitoring system (CGMS). Diabet Metab. 2003;29:608-12.

16. Steil GM, Rebrin K, Mastrototaro J, Bernaba B, Saad MF. Determination of plasma glucose during rapid glucose excursions with a subcutaneous glucose sensor. Diabetes Technol Ther. 2003;5(1):27-31.

17. Yates K, Hasnat Milton A, Dear K, Ambler G. Continuous glucose monitoring-guided insulin adjustment in children and adolescents on near-physiological insulin regimens: a randomized controlled trial. Diabetes Care. 2006;29:1512-7.

18. Lagarde WH, Barrows FP, Davenport ML, Kang M, Guess HA, Calikoglu AS. Continuous subcutaneous glucose monitoring in children with type 1 diabetes mellitus: a single-blind, randomized, controlled trial. Pediatr Diabetes. 2006;7:159-64.

19. McGowan K, Tomas W, Moran A. Spurius reporting of nocturnal hypoglycemia by CGMS in patients with thigthtyle controlled diabetes. Diabetes Care. 2002;25:1499-1503.

20. Boland E, Monsod T, Delucia M, Brandt CA, Fernando S, Tamborlane VW. Limitations of conventional methods of self-monitoring of blood glucose: lessons learned from 3 days of continuous glucose sensing in pediatric patients with type 1 diabetes. Diabetes Care. 2001;24(11):1858-62.
21. Chico A, Vidal-Rios P, Subira M, Novials A. The continuous glucose monitoring system is useful for detecting unrecognized hypoglycemias in patients with type 1 and type 2 diabetes but is not better than frequent capillary glucose measurements for improving metabolic control. Diabetes Care. 2003;26:1153-7.

22. Guillod L, Comte-Perret S, Monbaron D, Gaillard RC, Ruiz J. Nocturnal hypoglycaemias in type 1 diabetic patients: what can we learn with continuous glucose monitoring? Diabetes Metab. 2007;33(5):360-5.

23. Jeha GS, Karaviti PL, Anderson B, et al. Continuous glucose monitoring and the reality metabolic control in preschool children with type 1 diabetes. Diabetes Care. 2004;27:2881-6.

Endereço para correspondência:

Frederico Fernandes Ribeiro Maia

Rua Nunes Vieira, 299, apto.702 - Santo Antônio

30350-120, Belo Horizonte MG

E-mail: fredfrm@hotmail.com/frederico@diabetes.med.br 